\title{
Insulin: still a miracle after all these years
}

\author{
Fabrizio Barbetti ${ }^{1}$ and Simeon I. Taylor ${ }^{2}$
}

'Bambino Gesù Children's Hospital IRCCS, Rome, Italy. 'Division of Endocrinology, Diabetes, and Nutrition, Department of Medicine, University of Maryland School of Medicine, Baltimore, Maryland, USA.

\begin{abstract}
The discovery of insulin almost 100 years ago has resulted in a remarkable increase in lifespan and quality of life for patients with type 1 diabetes. The Joslin Medalist Study has allowed researchers to access and study patients (Medalists) with type 1 diabetes who have been insulin dependent for 50 years or more. In this issue of the $J C l$, Yu et al. evaluated HLA variants, autoantibody status, $\boldsymbol{\beta}$ cell function, $\mathrm{C}$-peptide release, and monogenetic diabetes genes in a cohort of Medalists. Postmortem analysis of pancreata from Medalists revealed the presence of insulin-positive $\beta$ cells in these patients. Moreover, some patients were still able to respond to metabolic stimuli despite long-term insulin dependence. Overall, the Medalist cohort was highly heterogenous, and genetic testing suggested that several patients would fall into categories other than type 1 diabetes on the basis of REVEL (rare exome variant ensemble learner) classification and may be able to transfer to other therapy options.
\end{abstract}

\section{Ground-breaking discovery: insulin}

As we approach the 100th anniversary of the discovery of insulin (1), we give thanks for the miraculous transformation of patients' lives resulting from that historical achievement of translational research. As reviewed by Dr. Elliott Joslin in 1917, two-thirds of children with type 1 diabetes died within 1.4 years after diagnosis $(2,3)$. A century later, as a result of the availability of insulin in combination with other technological advances, patients with type 1 diabetes can look forward to near-normal longevity (4). Prior to the discovery of insulin, the prevalence of type 1 diabetes was estimated to be approximately 5 cases/100,000 individuals in the United States (5). Currently, the prevalence of type 1 diabetes is now almost 100-fold higher (i.e., 400 cases/ 100,000 individuals); however, this is pri- marily because of the miraculous ability of insulin to extend life.

\section{The Medalists}

In this issue of the JCI, Yu et al. (6) from the Joslin Diabetes Center characterize 1018 Joslin Medalists, all of whom have lived for at least 50 years after being diagnosed with type 1 diabetes. Viewed from a historical perspective, it is a truly remarkable achievement for a single center to have conducted thorough investigations of such a large number of patients with type 1 diabetes and such impressive longevity. Approximately $18 \%$ of these research subjects underwent two evaluations separated by four years, providing further insight into the natural history of the disease. The Joslin Medalist population is enriched with patients who have had above-average clinical outcomes. Many factors may have contributed to

Related Article: p. 3252

Conflict of interest: SIT serves as a paid consultant for lonis Pharmaceuticals and is an inventor on patents covering the use of metreleptin as a treatment for lipodystrophy (patent number US8318666B2) and the use of fusion proteins containing fibronectin-derived serum albumin-binding domains to extend the pharmacokinetics of therapeutic proteins (patent number US9540424B2).

Copyright: (c) 2019, American Society for Clinical Investigation.

Reference information: / Clin Invest. 2019;129(8):3045-3047. https://doi.org/10.1172/JCI130310.

these good outcomes, including, for example, access to high-quality medical care, adherence to state-of-the-art therapeutic regimens, and favorable genetics.

$\mathrm{Yu}$ and colleagues emphasize several conclusions with respect to $\beta$ cell function (6). Despite more than 50 years of autoimmune attack, insulin-positive $\beta$ cells were detected by immunohistopathology in all 68 patients who underwent postmortem analysis of the pancreas. Among the 516 research subjects who underwent a mixed-meal tolerance test (MMTT), approximately 6\% exhibited an increase in C-peptide levels in response to a mixed meal. However, the presence or absence of a C-peptide response was not associated with clinical characteristics, such as glycated hemoglobin A1c (HbA1c) or the prevalence of diabetic complications. Thirty research subjects with baseline C-peptide levels greater than or equal to $0.1 \mathrm{ng} / \mathrm{mL}$ underwent hyperglycemic clamps with arginine infusion. Almost half of these research subjects experienced at least a doubling of C-peptide levels in response to glucose and/or arginine. The authors emphasized the possibility that the small number of residual functioning $\beta$ cells, which had escaped autoimmune destruction, could somehow be "rejuvenated," with the hope of restoring insulin secretion even after decades of type 1 diabetes.

In many respects, these Joslin Medalists had many features typical of patients with type 1 diabetes (6). For example, 93.5\% of the patients possessed the DR3 or DR4 HLA risk alleles, and $43.6 \%$ of the Medalists were positive for autoantibodies directed against either tyrosine phosphatase-like insulinoma antigen (IA2) or glutamic acid decarboxylase (GAD). Interestingly, patients with undetectable C-peptide levels were more likely to have GAD autoantibodies, whereas patients with detectable C-peptide were almost twice as likely to have IA2 autoantibodies. Patients who responded to a MMTT had lower titers of autoantibodies. As might have been predicted, patients who 
were negative for both autoantibodies and HLA risk alleles (DR3 and DR4) had the best preserved $\beta$ cell function, with $\mathrm{C}$-peptide levels that responded to both glucose and arginine.

Yu et al. (6) present thought-provoking observations with respect to genetic heterogeneity among the Joslin Medalists enrolled in their study. In 2003, an expert panel convened by the American Diabetes Association established an etiologic classification of diabetes (7). In addition to the common forms of diabetes (type 1 and type 2 diabetes), the panel introduced a category of diabetes, the so-called "genetic defects of $\beta$ cell function," that includes maturity-onset diabetes of the young (MODY), neonatal diabetes, and a number of syndromic forms of diabetes. The panel also defined a category termed "genetic defects of insulin action" that includes inter alia syndromes due to mutations in the insulin receptor gene. The true prevalence of monogenic diabetes is currently unknown, but recent studies showed that MODY alone or MODY together with other monogenic forms accounts for at least $6 \%$ of pediatric patients referred for diabetes or abnormal glucose tolerance (8, 9). Patients with MODY are usually lean and are often misdiagnosed as having type 1 diabetes. However, it is becoming increasingly evident that a sizeable number of pediatric patients with MODY may present with obesity (10) and can be misclassified as type 2 diabetes.

\section{Identification of monogenetic variants}

Yu et al. (6) studied patients with type 1 diabetes who received their 50-year Joslin Medals prior to 2018 and who were first diagnosed prior to 1968. Individuals who received Medals between 1998 and 2003 (first diagnosis prior to 1953) were also invited to participate in the study. In short, these research subjects were first diagnosed prior to the modern appreciation of the existence of genetic defects in $\beta$ cell function or insulin action. Accordingly, Yu et al. (6) inquired whether some of their research subjects might harbor genetic variants in known monogenic diabetes genes. Interestingly, rare variants in monogenic diabetes genes were identified in $27.5 \%$ of Medalists. However, multiple lines of evidence are required to provide rigorous proof that a genetic variant contributes to disease pathogenesis - for example, experiments demonstrating that the genetic variant alters protein function as well as genetic evidence that a variant cosegregates with signs and symptoms of disease. Because it was impractical to provide this type of rigorous evidence for all 280 Joslin Medalists in whom genetic variants were identified, Yu et al. applied an in silico technique based on REVEL (rare exome variant ensemble learner) scores (11). Using a REVEL score cutoff above 0.75 (52.1\% sensitivity and 96.7\% specificity), Yu et al. (6) identified heterozygous variants they viewed as likely contributors to disease pathogenesis in $7.9 \%$ of the 1018 Medalist patients, all of whom had the diagnosis of type 1 diabetes. Most of these variants would be classified as variants of uncertain significance and/or located in a recessive diabetes gene. However, some variants have previously been described in the literature as being responsible for autosomal-dominant diabetes, and a functional effect has been demonstrated directly using validated experimental approaches. These data have potential implications for screening strategies to identify patients with genetic variants in monogenic diabetes genes. The presence or absence of HLA risk alleles appeared to be predictive, with $18.3 \%$ of HLA-negative patients harboring variants in monogenic diabetes genes compared with only $7.4 \%$ of patients who were positive for HLA-DR3 or HLD-DR4. However, the presence or absence of autoantibodies did not appear to be highly predictive, with genetic variants being identified in monogenic diabetes genes in $7.3 \%$ of autoantibody-positive subjects versus $8.7 \%$ of autoantibody-negative research subjects. The highest C-peptide levels were observed in patients who were negative for HLA risk alleles and negative for autoantibodies, but positive for variants in monogenic diabetes genes (median random C-peptide levels of $0.80 \mathrm{ng} / \mathrm{ml}$ versus $0.05 \mathrm{ng} / \mathrm{ml}$ for pooled data derived from all other subgroups). Taken together, these data raise the possibility that some patients may have been incorrectly tagged with the diagnosis of type 1 diabetes and would be more correctly classified as having a form of monogenic diabetes.

\section{Concluding remarks}

It is tempting to extrapolate from these data to define an approach to screen for the presence of monogenic diabetes variants in patients with a diagnosis of type 1 diabetes. Given the data from this Joslin Medalist study, such genetic screening is most likely to be productive in patients who are negative for HLA risk alleles (HLA-DR3 and HLA-DR4). Furthermore, as exemplified by research subjects 2,3 , and 7 , who carry pathogenic mutations (see Table 5 in Yu et al. and ref. 6), the diagnosis of MODY should be suspected in patients diagnosed with type 1 diabetes who are negative for the type 1 diabetes autoantibody and also show detectable C-peptide levels during fasting that increase after a MMTT. The absence of vertical transmission of diabetes does not exclude the possibility that diabetes may be caused by a variant in a monogenic diabetes gene. Nevertheless, because these conclusions are based on relatively small numbers of patients in the relevant subgroups, the suggested screening strategies are offered tentatively and will require validation in larger groups of patients. Future follow-up studies could be conducted in these Joslin Medalist patients to elucidate the functional impact of variants of uncertain significance found in genes associated with dominantly inherited forms of monogenic diabetes.

Finally, there are numerous examples of patients with neonatal diabetes due to KCNJ11 or ABCC8 mutations who were successfully transferred to sulfonylureas after 25 or more years of insulin therapy (12-14). Thus, it is possible that some of these Medalist patients could be effectively treated with sulfonylureas, such as those with a genetic diagnosis of HNF1A MODY 3 (at least 4 patients in this study) or carriers of variants in KCNJ11, ABCC8, and HNF4A. It would be a remarkable outcome if identification of genetic variants in monogenic diabetes genes enabled a subpopulation of Medalist patients to switch to an oral drug rather than insulin. Despite the fact that these patients have done extremely well using insulin for more than 50 years, insulin therapy requires considerable effort on the part of patients. Therefore, when it is possible to achieve good metabolic control with a sulfonylurea, patients with monogenic diabetes tend to welcome the change to an oral drug.

\section{Acknowledgments}

SIT acknowledges support from the National Institute of Diabetes and Diges- 
tive and Kidney Diseases (NIDDK) (P30DK072488 and R0118942).

Address correspondence to: Simeon I. Taylor, University of Maryland School of Medicine, HSF-III, Room 4182, 655 West Baltimore Street, Baltimore, Maryland 21201, USA.Email: staylor2@som.umaryland.edu.

1. Banting FG, Best CH, Collip JB, Campbell WR, Fletcher AA. Pancreatic Extracts in the Treatment of Diabetes Mellitus. Can Med Assoc J. 1922;12(3):141-146.

2. Joslin EP. The Treatment of Diabetes Mellitus, with Observations upon 1300 cases. Philadelphia: Lea and Febiger; 1917.

3. Gale EAM. Natural history. Diapedia 2014; 2104085199 rev. no. 27. Website. https://doi. org/10.14496/dia.2104085199.27. Accessed June 26, 2019.

4. Livingstone SJ, et al. Estimated life expectancy in a Scottish cohort with type 1 diabetes, 20082010. JAMA. 2015;313(1):37-44.

5. Gale EA. The rise of childhood type 1 diabetes in the 20th century. Diabetes. 2002;51(12):3353-3361.

6. Yu MG, Keenan HA, Shah HS, et al. Residual $\beta$ cell function and monogenic variants in longduration type 1 diabetes patients. J Clin Invest. 2019;129(8):3252-3263.

7. Expert Committee on the Diagnosis Classification of Diabetes Mellitus. Report of the expert committee on the diagnosis and classification of diabetes mellitus. Diabetes Care. 2003;26 Suppl 1:S5-20.

8. Johansson BB, et al. Targeted next-generation sequencing reveals MODY in up to $6.5 \%$ of antibody-negative diabetes cases listed in the Norwegian Childhood Diabetes Registry. Diabetologia. 2017;60(4):625-635.

9. Delvecchio M, et al. Monogenic Diabetes Accounts for $6.3 \%$ of Cases Referred to 15 Italian Pediatric Diabetes Centers During 2007 to 2012 J Clin Endocrinol Metab. 2017;102(6):1826-1834.

10. Kleinberger JW, et al. Monogenic diabetes in overweight and obese youth diagnosed with type 2 diabetes: the TODAY clinical trial. Genet Med. 2018;20(6):583-590.

11. Ioannidis NM, et al. REVEL: An Ensemble Method for Predicting the Pathogenicity of Rare Missense Variants. Am J Hum Genet. 2016;99(4):877-885.

12. Koster JC, Cadario F, Peruzzi C, Colombo C, Nichols CG, Barbetti F. The G53D mutation in Kir6.2 (KCNJ11) is associated with neonatal diabetes and motor dysfunction in adulthood that is improved with sulfonylurea therapy. JClin Endocrinol Metab. 2008;93(3):1054-1061.

13. Bowman P, et al. Effectiveness and safety of long-term treatment with sulfonylureas in patients with neonatal diabetes due to KCNJ11 mutations: an international cohort study. Lancet Diabetes Endocrinol. 2018;6(8):637-646.

14. Pearson ER, Starkey BJ, Powell RJ, Gribble FM, Clark PM, Hattersley AT. Genetic cause of hyperglycaemia and response to treatment in diabetes. Lancet. 2003;362(9392):1275-1281. 